\title{
Vanadium Dioxide as a Natural Disordered Metamaterial: Perfect Thermal Emission and Large Broadband Negative Differential Thermal Emittance
}

\author{
Mikhail A. Kats, Romain Blanchard, Shuyan Zhang, Patrice Genevet, \\ Changhyun Ko, Shriram Ramanathan, and Federico Capasso* \\ School of Engineering and Applied Sciences, Harvard University, Cambridge, Massachusetts 02138, USA
}

(Received 24 April 2013; published 21 October 2013)

\begin{abstract}
We experimentally demonstrate that a thin (approximately 150-nm) film of vanadium dioxide $\left(\mathrm{VO}_{2}\right)$ deposited on sapphire has an anomalous thermal emittance profile when heated, which arises because of the optical interaction between the film and the substrate when the $\mathrm{VO}_{2}$ is at an intermediate state of its insulator-metal transition (IMT). Within the IMT region, the $\mathrm{VO}_{2}$ film comprises nanoscale islands of the metal and dielectric phases and can thus be viewed as a natural, disordered metamaterial. This structure displays "perfect" blackbodylike thermal emissivity over a narrow wavelength range (approximately $40 \mathrm{~cm}^{-1}$ ), surpassing the emissivity of our black-soot reference. We observe large broadband negative differential thermal emittance over a $>10^{\circ} \mathrm{C}$ range: Upon heating, the $\mathrm{VO}_{2}$-sapphire structure emits less thermal radiation and appears colder on an infrared camera. Our experimental approach allows for a direct measurement and extraction of wavelength- and temperature-dependent thermal emittance. We anticipate that emissivity engineering with thin-film geometries comprising $\mathrm{VO}_{2}$ and other thermochromic materials will find applications in infrared camouflage, thermal regulation, and infrared tagging and labeling.
\end{abstract}

DOI: 10.1103/PhysRevX.3.041004

Thermal radiation is light that is emitted by an object at a temperature above absolute zero. The spectrum and intensity of thermal radiation emitted by an object are a function of its temperature and emissivity, which is, in general, frequency $(f)$ dependent. This relation is expressed by

$$
I(K, T)=2 h c \frac{K^{3}}{e^{h c K / k_{B} T}-1} \varepsilon(K),
$$

where $I$ is the spectral radiance (or the spectral radiant energy density), $K=f / c$ is the spectroscopic wave number, $T$ is the temperature expressed in kelvin, $h$ is Planck's constant, $c$ is the speed of light in vacuum, $k_{b}$ is the Boltzmann constant, and $\varepsilon(K)$ is the frequency-dependent emissivity [1]. More specifically, the spectral radiance is the radiant power emitted from a unit area of the source per unit solid angle, in the wave-number interval from $K$ to $K d K$, and has units of $\mathrm{W} \mathrm{cm}^{-1}$. The factor in front of $\varepsilon(K)$ in Eq. (1) is known as Planck's law and describes blackbody emission. For most objects, $\varepsilon(K)$ is largely independent of temperature (or other external variables such as applied fields).

There is substantial interest in engineering $\varepsilon(K)$ for applications ranging from incandescent light sources [2] to heat management [3-5] to thermal tagging and imaging [6]. In determining $\varepsilon(K)$ for various materials and structures, frequent use is made of Kirchhoff's law of

\footnotetext{
*capasso@seas.harvard.edu
}

Published by the American Physical Society under the terms of the Creative Commons Attribution 3.0 License. Further distribution of this work must maintain attribution to the author(s) and the published article's title, journal citation, and DOI.
Subject Areas: Metamaterials, Optics, Optoelectronics

thermal radiation, which states that the emissivity of an object $\varepsilon(K)$ is equal to its frequency-dependent absorptivity $a(K)[1]$.

One approach to engineering $\varepsilon(K)$ has been to select materials with appropriate material dispersion to achieve selective thermal emission [7]. A complementary approach involves surface texturing, either disordered [2] or highly ordered in the case of gratings [8] or photonic crystals [9]. Similarly, photonic cavities can enhance or suppress thermal emission [10,11]. More recently, optical antennas [12] and metamaterials [13] have also been employed to tailor the directionality and spectrum of thermal emission.

In addition to these static schemes, certain tunable materials can be employed to dynamically manipulate $\varepsilon(K)$. Of particular interest are electrochromic materials such as tungsten oxide $\left(\mathrm{WO}_{3}\right)$, which undergoes significant change in optical and infrared properties under an applied voltage and can therefore be used to modulate the emissivity of thermal radiators for applications such as temperature control of satellites by radiative cooling [3,4].

Modulation of the emissivity can also be achieved by using thermochromic materials, whose optical properties are temperature dependent. Unlike in the case of electrochromic materials, a change in temperature can simultaneously alter the emissivity $\varepsilon(K, T)$ of the object incorporating a thermochromic material, and the blackbody contribution to the spectral radiance $K^{3} /\left(e^{h c K / k_{B} T}-1\right)$ [see Eq. (1)]. A potential benefit of tuning based on thermochromic materials is that it allows for passive "smart" devices that can operate without the need for external power or controls. For example, a radiator that has low emissivity at low temperatures and high emissivity at high 
temperatures can help keep heat in when cold and radiate heat away faster when hot, making it useful for passively maintaining a desired temperature [5].

A promising thermochromic material is vanadium dioxide $\left(\mathrm{VO}_{2}\right)$, a correlated oxide that experiences a thermally induced insulator-metal transition (IMT) near room temperature $\left(T_{c} \sim 67{ }^{\circ} \mathrm{C}\right.$ in bulk crystals), which takes the material from an insulating state to a metallic one. The IMT, which can also be triggered electrically and optically, is the target of research for the realization of a variety of electronic switching devices [14] and also finds uses in optical switching [15]. The present literature on tuning an object's thermal emissivity using $\mathrm{VO}_{2}$ has largely focused on the considerable change of infrared optical properties between the extreme states of the phase transition-fully insulating and fully metallic [5,16-19]. However, very rich physics can be found within the transition region itself, which can be harnessed to obtain additional control over thermal emission properties.

Probing the physics of this intermediate state is still at an early stage because the material quality of $\mathrm{VO}_{2}$ films has only recently been improved to the point where a complex region such as the intermediate state of the phase transition can be explored with reproducibility. The IMT in this material has been known for over 50 years; however, bulk crystals often crack during the transition because of stress (as is often the case for brittle ceramics) [20]. Thinfilm structures can be made more robust to the stress relaxation caused by substrate clamping; however, careful thermal profiles and composition control are required to obtain reversible transition properties $[21,22]$. Studies on thin-film $\mathrm{VO}_{2}$ with reproducible phase-transition properties can be considered an emerging field, and within that, the intermediate state (i.e., a mixture of metallic and insulating states) is now being recognized as an opportunity, especially given recent interest in metamaterials that often require composite structures comprising domains of subwavelength dimensions with vastly different optical properties [23].

In this article, we show that a geometry comprising a thin film of $\mathrm{VO}_{2}$ on a sapphire substrate can exhibit "perfect" blackbodylike emissivity (approximately 1) over a narrow range of frequencies when the $\mathrm{VO}_{2}$ is in its transitional state and operates as a natural, tunable metamaterial, i.e., an effective medium with widely tunable infrared optical properties. As a result of this resonance in emissivity, the sample displays substantial broadband negative differential thermal emittance; i.e., as the sample is heated, the thermal emission decreases.

In $\mathrm{VO}_{2}$ thin films, the IMT occurs gradually with increasing temperature: Nanoscale inclusions of the metallic phase emerge in the surrounding insulating-phase $\mathrm{VO}_{2}$, which grow and connect in a percolation process, eventually leading to a fully metallic state at the end of the transition [24,25]. These metallic inclusions are much smaller than the scale of the wavelength at infrared frequencies, and thus $\mathrm{VO}_{2}$ can be viewed as a natural, reconfigurable, disordered metamaterial with variable effective optical properties across the phase transition. In Ref. [26], the authors utilized this unique temperature-dependent dispersion of the effective medium to demonstrate that a film of $\mathrm{VO}_{2}$ with thickness much smaller than the wavelength deposited on sapphire can operate as a temperaturetunable absorber; in particular, nearly perfect absorption was achieved at a particular temperature for a narrow range of infrared wavelengths. The reflectivity of such a device varies dramatically and nonmonotonically across the phase transition, with the strong absorption feature appearing during an intermediate state of $\mathrm{VO}_{2}$ as a result of critical coupling to an "ultra-thin-film resonance"; similar resonances have also recently been demonstrated using semiconductor films on metallic substrates or Bragg reflectors in the visible for tailoring reflectivity and absorption [27-30], and semiconductor films on sapphire or highly doped semiconductor substrates for tailoring absorption and thermal emission [31,32]. Since $\varepsilon(K)=a(K)$, such a thin-film $\mathrm{VO}_{2}$-sapphire structure is expected to have an emissivity $\varepsilon(K, T)$ that also depends strongly and nonmonotonically on temperature.

Our sample consists of an epitaxial $\mathrm{VO}_{2}$ film of approximately $150 \mathrm{~nm}$ in thickness, grown on a polished singlecrystal $c$-plane sapphire by rf-magnetron sputtering with a $\mathrm{V}_{2} \mathrm{O}_{5}$ target (99.9\% purity, AJA International, Inc.). During film growth, the substrate temperature and rf source gun power are kept constant at $550{ }^{\circ} \mathrm{C}$ and $125 \mathrm{~W}$, respectively. A mixture of $99.50-\mathrm{sccm} \mathrm{Ar}$ and $0.50-\mathrm{sccm}$ $\mathrm{O}_{2}$ is used as the sputtering gas, maintaining the total pressure at 10 mTorr.

We measure the thermal emission from our $\mathrm{VO}_{2}$-sapphire sample by mounting it on a temperaturecontrolled stage (Bruker A599), changing the temperature from $40^{\circ} \mathrm{C}$ to $100^{\circ} \mathrm{C}$ and back down (resolution of $0.5^{\circ}$ in the range of $55^{\circ} \mathrm{C}-85^{\circ} \mathrm{C}$ and $5^{\circ}$ outside of that, waiting at least $60 \mathrm{~s}$ to allow the temperature to settle), and directly sending the emitted light into a nitrogen-purged Fourier transform infrared (FTIR) spectrometer (Bruker Vertex 70) equipped with a room-temperature doped triglycine sulfate (DTGS) detector (Fig. 1). As a reference, we replace the sample with black soot [1], deposited onto a gold-coated silicon wafer using a candle (deposition time $>10 \mathrm{~min}$ ). After deposition, the soot-coated wafer is baked at $200{ }^{\circ} \mathrm{C}$ for $30 \mathrm{~min}$ to remove any excess paraffin from the candle. At moderate temperatures, candle-deposited soot is expected to have a wavelength-independent emissivity $\varepsilon$ between 0.95 and 0.98 in the infrared [33-35]; for this work, we assume $\varepsilon=0.96$. Midinfrared reflectance measurements taken using a microscope (Bruker Hyperion 2000 , numerical aperture $=0.4,15 \times$ objective) confirm that the soot sample is a good blackbody reference (reflectance $<0.01$ with some light presumed to be scattered; data are not shown). 
Fourier transform infrared spectrometer

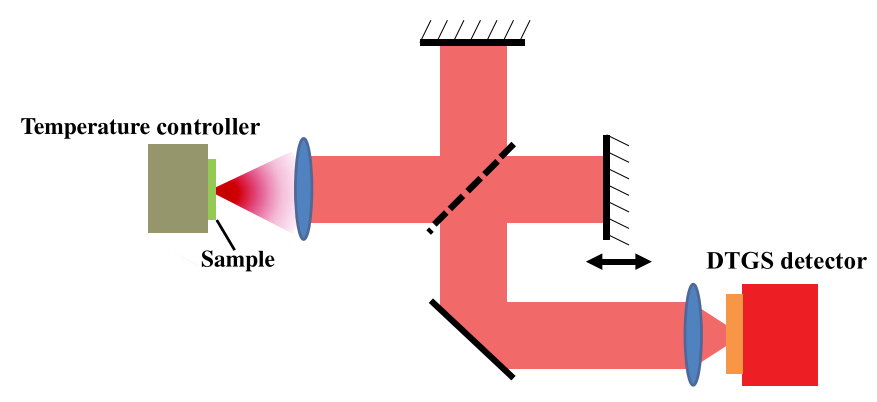

FIG. 1. Experimental setup. The $\mathrm{VO}_{2}$-sapphire sample is mounted on a temperature-controlled stage, and the thermal emission is sent into an FTIR spectrometer equipped with a DTGS detector.

To obtain an accurate emission spectrum, we have to correct for the wave-number-dependent response of the optics of the FTIR spectrometer and the detector and also account for the thermal emission from sources other than our sample [1,36]. In particular, both the detector and the optical components of the FTIR (the beam splitter, mirrors, etc.) are all at room temperature, and all emit thermal radiation. Accounting for these contributions becomes more complicated in our case, compared to more conventional emittance measurements, because our sample is partially reflective, and the reflectivity changes significantly as a function of temperature, which affects how much of the thermal radiation emitted by the detector returns back to the detector.

A particular temperature-dependent measured spectrum $S(K, T)$ can be broken down as

$$
\begin{aligned}
S(K, T)= & m(K, T)\left\{I(K, T)+b_{\text {instr }}(K)\right. \\
& \left.-[1-R(K, T)] b_{\text {det }}(K)\right\},
\end{aligned}
$$

where $I(K, T)$ is the actual spectral radiance of the sample; $m(K, T)$ is the instrument response transfer function, including the effects of atmospheric absorption along the light path between the sample and the detector; $b_{\text {instr }}(K)$ is the thermal contribution of the instrument, including emission from the optics and the walls of the FTIR spectrometer (but not from the detector); $b_{\operatorname{det}}(K)$ is the thermal emission from the detector; and $R(K, T)$ is the reflectivity of the sample that can be temperature dependent for thermochromic materials. In our measurement, we assume that $b_{\text {instr }}(K)=0$ because our DTGS detector is at the same temperature as the rest of the instrument, so there is no net flow of thermal radiation between the detector and the optics and walls. There is substantial radiation from the detector itself, so $b_{\text {det }}(K)$ cannot be neglected, and a portion of this radiation that enters the interferometer and reaches the sample is reflected back toward the detector; this contribution is accounted for by the $[1-R(K, T)]$ term in Eq. (2).
Because both our black-soot reference and $\mathrm{VO}_{2}$-sapphire sample are opaque within the wavelength range of interest (within the 5- to $15-\mu \mathrm{m}$ range, sapphire is opaque as a result of multiple phonon resonances that are present [26,37]), we can write the emissivity as $\varepsilon(K, T)=$ $a(K, T)=1-R(K, T)$, which simplifies Eq. (2) to

$$
S(K, T)=m(K, T) \varepsilon(K, T)\left[I_{\mathrm{BB}}(K, T)-b_{\mathrm{det}}(K)\right],
$$

where $I_{\mathrm{BB}}(K, T)$ is the thermal radiation spectrum from a perfect blackbody. Note that it is important to select the appropriate units when representing the Planck distribution function $I_{\mathrm{BB}}(K, T)$, as the expressions differ depending on which units are used, e.g., wave number or wavelength. Since an FTIR yields spectra with constant resolution in wave number [1], we choose to use spectroscopic wavenumber units (which are equivalent to frequency units since $K=f / c)$.

Given a reference sample with a known emissivity such as the black soot, one can calculate $m(K, T)$ and $b_{\text {det }}(K)$ by measuring the emitted spectrum at two different temperatures $T_{1}$ and $T_{2}$, and by solving the system of two equations. In our measurement, however, this calculation is unnecessary because $\varepsilon(K, T)$ is factored out in Eq. (3). Instead, we can note that given the measured spectra $S_{\text {sample }}(K, T)$ and $S_{\text {ref }}(K, T)$ from our sample and reference, respectively, and knowledge of the reference emissivity $\varepsilon_{\text {ref }}(K, T)$, we can immediately obtain $\varepsilon_{\text {sample }}(K, T)$ by

$$
\frac{S_{\text {sample }}(K, T)}{S_{\text {ref }}(K, T)}=\frac{\varepsilon_{\text {sample }}(K, T)}{\varepsilon_{\text {ref }}(K, T)} .
$$

This analysis method is applicable only to samples that are smooth (any roughness must be on a substantially smaller scale than the wavelength of emitted light), which is the case for our $\mathrm{VO}_{2}$-sapphire sample. For rough samples, not all of the light emitted by the detector will be specularly reflected from the sample, and instead some thermal emission from the surrounding area may be scattered into the beam path by the sample; in this case, extra care must be taken during data analysis.

We use Eq. (4) to determine the experimental emissivity of our $\mathrm{VO}_{2}$-sapphire sample, which is plotted for increasing temperatures in Figs. 2(a) and 2(b). From the experimental emissivity, we calculate the spectral radiance $I(K, T)=$ $\varepsilon(K, T) I_{\mathrm{BB}}(K, T)$ of our black-soot reference and the $\mathrm{VO}_{2}$-sapphire sample, shown in Fig. 2(c), for three different temperatures. It can be directly observed that while the thermal emission from the black-soot reference is monotonically increasing with increasing temperature, the emission from the $\mathrm{VO}_{2}$-sapphire sample first increases and then decreases. At a particular temperature (approximately $74.5^{\circ} \mathrm{C}$ ) and wavelength (approximately $864 \mathrm{~cm}^{-1}$ ), the emissivity approaches unity (Fig. 2), indicating that at that wavelength, the sample displays nearly perfect blackbodylike emission, corresponding to the "perfect absorption" condition demonstrated in Ref. [26], which is the result of 

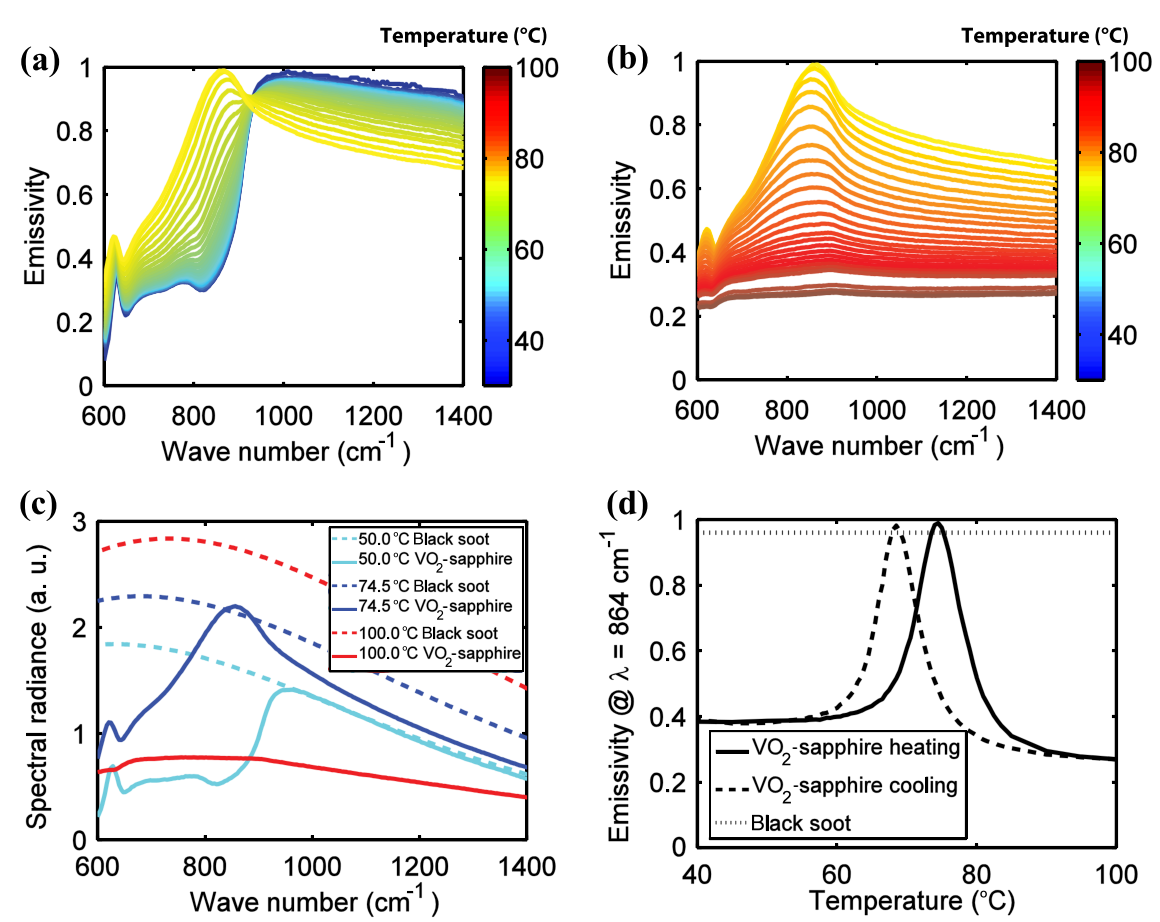

FIG. 2. (a),(b) Experimentally determined evolution of the $\mathrm{VO}_{2}$-sapphire emissivity for increasing temperature, separated into ranges of $35^{\circ} \mathrm{C}-74.5^{\circ} \mathrm{C}$ and $74.5^{\circ} \mathrm{C}-100{ }^{\circ} \mathrm{C}$ for visual clarity. (c) Thermal emission density (spectral radiance) from black soot (dashed lines) and our $\mathrm{VO}_{2}$-sapphire sample (solid lines) for three different temperatures. The data were taken for increasing temperatures. (d) Thermal emissivity of the $\mathrm{VO}_{2}$-sapphire sample at a wave number of $864 \mathrm{~cm}^{-1}$ for heating (solid line) and cooling (dashed line), respectively. The dotted line denotes the assumed emissivity of the black-soot reference $(\varepsilon=0.96)$.

critical coupling to a strong absorption resonance in the film. The peak in infrared emissivity is relatively broadband (approximately $200 \mathrm{~cm}^{-1}$ ), with the emissivity surpassing that of the black-soot reference between 840 and $885 \mathrm{~cm}^{-1}$. The spectral position of this emissivity peak can be tuned from approximately 950 to $770 \mathrm{~cm}^{-1}$ by varying the $\mathrm{VO}_{2}$ film thickness from approximately 20 to $400 \mathrm{~nm}$ [26]. The emissivity exhibits hysteresis in temperature [Fig. 2(c)] due to the intrinsic hysteresis in undoped $\mathrm{VO}_{2}$ [14]. Note that the data in Figs. 2(a) and 2(b) are shown for increasing temperatures only.

We integrate $I(K, T)$ of the black soot and $\mathrm{VO}_{2}$-sapphire samples over the 8- to $14-\mu \mathrm{m}$ atmospheric transparency window and plot it as a function of temperature in Fig. 3(a). Plotted this way, it is clear that while heating, the samples display broadband negative differential thermal emittance over the $73^{\circ} \mathrm{C}-85^{\circ} \mathrm{C}$ temperature range while heating and over $68^{\circ} \mathrm{C}-80^{\circ} \mathrm{C}$ while cooling. The magnitude of the effect is large: Over an approximately $10^{\circ} \mathrm{C}$ temperature range, the slope is even larger in magnitude than the blackbody slope, indicating that the $\mathrm{VO}_{2}$-sapphire sample has a larger negative differential thermal emittance than the blackbody positive differential thermal emittance over the same temperature range. Imaging these samples with a thermal camera (FLIR Systems Thermovision A40) confirms that because of the negative differential thermal emittance, the sample appears cooler even as it is heating up [Fig. 3(c)]. Using the camera, some inhomogeneities in the thermal emittance of the film are visible that likely result from slight inhomogeneities in the temperature and gradual long-range variations in the film thickness and roughness; these emittance inhomogeneities are amplified around the phase-transition temperature.

The $\mathrm{VO}_{2}$-sapphire thin-film geometry (and, more generally, any geometries incorporating $\mathrm{VO}_{2}$ with optical resonances in the infrared) is promising for a wide array of applications calling for tunable infrared emissivity, narrow-band perfect blackbodylike emission, negative differential thermal emittance, emissivity hysteresis, or some combination thereof. As one example, the emittance profile shown in Fig. 3 can be utilized to make a rewritable infrared "blackboard" by keeping the entire sample at the phase-transition temperature and using a cold or hot probe (such as a laser beam or soldering iron) to "write" messages by locally changing the emissivity. These persistent messages could be viewed with a thermal camera but would otherwise be invisible. A digital version of this device can be used as a rewritable infrared identification tag. As another example, the structure can be used as a type of infrared camouflage: Within the $85^{\circ} \mathrm{C}$ to $100^{\circ} \mathrm{C}$ temperature region, the total thermally emitted power remains roughly constant, and therefore an infrared camera would not be sensitive to changes in temperature. The width of this flat-emittance region can be extended by 

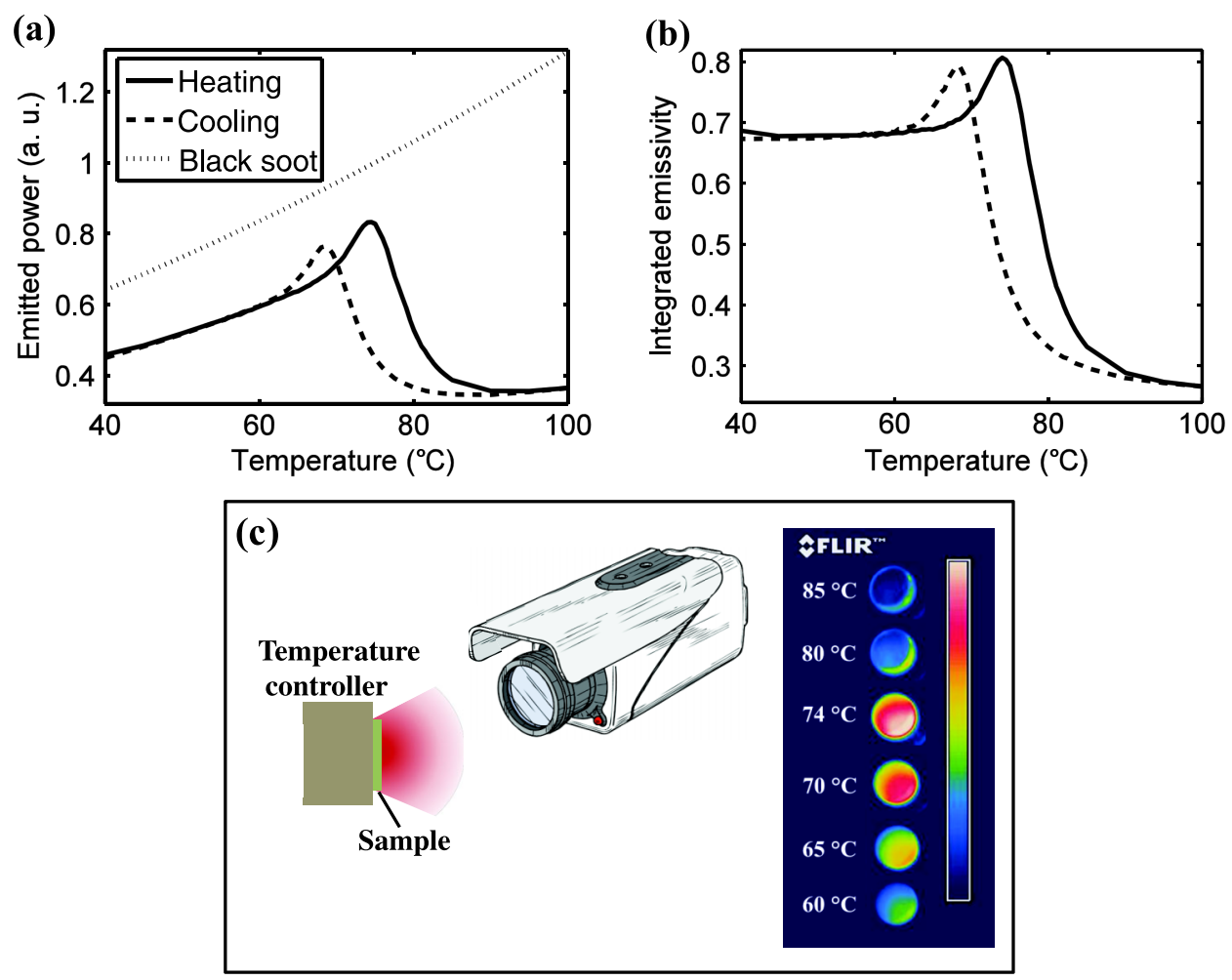

FIG. 3. (a) Emitted power of the $\mathrm{VO}_{2}$-sapphire sample integrated over the 8- to $14-\mu \mathrm{m}$ atmospheric transmission window for heating (solid line) and cooling (dashed line), compared to the emitted power from black soot. (b) The integrated emissivity of the $\mathrm{VO}_{2}$-sapphire sample over the 8- to $14-\mu \mathrm{m}$ wavelength range. (c). Infrared camera images of the sample $($ diameter $=1 \mathrm{~cm})$ for increasing temperatures.

decreasing the sharpness of the phase transition, which can be accomplished by introducing defects into the $\mathrm{VO}_{2}$ films [38].

Depending on the application, the hysteresis intrinsic to $\mathrm{VO}_{2}$ can either be beneficial (as in memory devices) or detrimental (for devices which require fast on and off switching). Fortunately, a variety of methods to modulate the hysteresis width have been studied, including engineering of the size and shape of grain boundaries [38] and stresses [39], as well as the introduction of various metallic dopants [40,41]. The aforementioned approaches have also been used to tailor the transition temperature of $\mathrm{VO}_{2}$ within the $0{ }^{\circ} \mathrm{C}$ to $100{ }^{\circ} \mathrm{C}$ range [38-41], further expanding the application space.

While our study is limited to thin films of $\mathrm{VO}_{2}$ on a sapphire substrate in the spectral region where the complex refractive index of sapphire is similar to that of metals at visible or UV wavelengths due to the presence of strong phonon resonances, a variety of other substrates can be used to engineer the wavelength of the peak in emissivity resulting from the ultra-thin-film resonance in $\mathrm{VO}_{2}$. Both highly doped semiconductors and transparent conducting oxides have been shown to possess complex refractive indices similar to that of sapphire at $\lambda=10-15 \mu \mathrm{m}$ (or metals in the visible or UV), and both can be engineered by modulating the doping [42] and are thus ideal candidate substrates for tailorable thermal emitters based on ultrathin films of $\mathrm{VO}_{2}$ or another material. The thin-film resonance condition can be further modified by introducing resonant metamaterials [43] or resonant optical antennas [44] on top of or within the ultrathin layer.

Ultimately, anomalous thermal emission properties that rely on the presence of a temperature-dependent emissivity such as the ones demonstrated in the present experiment can also be realized by integrating thermochromic materials into any number of optical structures, including photonic crystals $[9,45]$ or artificial metamaterials [13]. The inherent advantages of our thin-film geometry featuring a natural, disordered metamaterial within the $\mathrm{VO}_{2}$ phasetransition region are structural simplicity and ease of fabrication that allow for the creation of large-area anomalous thermal emitters. Nonetheless, emerging top-down and bottom-up fabrication methods may allow for the creation of large-area anomalous thermal emitters that combine thermochromics such as $\mathrm{VO}_{2}$ with photonic crystals and artificial metamaterials to provide additional design degrees of freedom.

In conclusion, we have experimentally studied the infrared thermal emittance of a structure comprising a deeply subwavelength thin film of vanadium dioxide $\left(\mathrm{VO}_{2}\right)$ on a sapphire substrate. Within the phase-transition region of its IMT, the $\mathrm{VO}_{2}$ film comprises nanoscale islands of 
insulator- and metal-phase $\mathrm{VO}_{2}$ that create a natural, disordered metamaterial with tunable optical dispersion and losses in the infrared, which leads to an absorption resonance within the film that appears and disappears upon temperature tuning. This resonance leads to a large peak in infrared emissivity spanning approximately $200 \mathrm{~cm}^{-1}$, including a $50-\mathrm{cm}^{-1}$ range over which the emissivity of the $\mathrm{VO}_{2}$-sapphire sample is greater than that of black soot, a commonly used blackbodylike emissivity reference. This emissivity peak remains significant even when the emittance spectrum is integrated over the 8- to $14-\mu \mathrm{m}$ atmospheric transparency window, and as a result, the sample also features a broad-temperature $\left(>10^{\circ} \mathrm{C}\right)$ region, over which it displays large negative differential thermal emittance such that the sample emits significantly less thermal radiation, even as it is heated up. These anomalous emittance properties can find uses in infrared camouflage, thermal regulation, infrared tagging and identification, and other applications.

We acknowledge helpful discussions with You Zhou, Sergey Shilov, Patrick Rauter, and Yu Yao, and financial support from the AFOSR under Grants No. FA9550-12-10289 and No. FA9550-08-1-0203. M. Kats is supported by the National Science Foundation through a Graduate Research Fellowship. S. Zhang is supported by the Singapore A*STAR National Science Fellowship.

[1] J. Mink, in Handbook of Vibrational Spectroscopy (Wiley, New York, 2006).

[2] A. Y. Vorobyev, V.S. Makin, and C. Guo, Brighter Light Sources from Black Metal: Significant Increase in Emission Efficiency of Incandescent Light Sources, Phys. Rev. Lett. 102, 234301 (2009).

[3] J.S. Hale, M. DeVries, B. Dworak, and J. A. Woollam, Visible and Infrared Optical Constants of Electrochromic Materials for Emissivity Modulation Applications, Thin Solid Films 313, 205 (1998).

[4] E. B. Franke, C. L. Trimble, M. Schubert, J. A. Woollam, and J.S. Hale, All-Solid-State Electrochromic Reflectance Device for Emittance Modulation in the Far-Infrared Spectral Region, Appl. Phys. Lett. 77, 930 (2000).

[5] M. Benkahoul, M. Chaker, J. Margot, E. Haddad, R. Kruzelecky, B. Wong, W. Jamroz, and P. Poinas, Thermochromic $\mathrm{VO}_{2}$ Film Deposited on Al with Tunable Thermal Emissivity for Space Applications, Solar Energy Mater. Sol. Cells 95, 3504 (2011).

[6] J. K. Coulter, C.F. Kelin, and J.C. Jafolla, Two Optical Methods for Vehicle Tagging, Proc. SPIE Int. Soc. Opt. Eng. 4708, 354 (2002).

[7] D. L. Chubb, A. T. Pal, M. O. Patton, and P. P. Jenkins, Rare Earth Doped High Temperature Ceramic Selective Emitters, J. Eur. Ceram. Soc. 19, 2551 (1999).

[8] J.-J. Greffet, R. Carminati, K. Joulain, J.-P. Mulet, S. Mainguy, and Y. Chen, Coherent Emission of
Light by Thermal Sources, Nature (London) 416, 61 (2002).

[9] M. U. Pralle, N. Moelders, M. P. McNeal, I. Puscasu, A. C. Greenwald, J. T. Daly, E. A. Johnson, T. George, D.S. Choi, I. El-Kady, and R. Biswas, Photonic Crystal Enhanced Narrow-Band Infrared Emitters, Appl. Phys. Lett. 81, 4685 (2002).

[10] N. J. Harrick and A. F. Turnet, A Thin Film Optical Cavity to Induce Absorption or Thermal Emission, Appl. Opt. 9, 2111 (1970).

[11] I. Celanovic, D. Perreault, and J. Kassakian, ResonantCavity Enhanced Thermal Emission, Phys. Rev. B 72, 075127 (2005).

[12] J. A. Schuller, T. Taubner, and M. L. Brongersma, Optical Antenna Thermal Emitters, Nat. Photonics 3, 658 (2009).

[13] X. Liu, T. Tyler, T. Starr, A. F. Starr, N. M. Jokerst, and W. J. Padilla, Taming the Blackbody with Infrared Metamaterials as Selective Thermal Emitters, Phys. Rev. Lett. 107, 045901 (2011).

[14] Z. Yang, C. Ko, and S. Ramanathan, Oxide Electronics Utilizing Ultrafast Metal-Insulator Transitions, Annu. Rev. Mater. Res. 41, 337 (2011).

[15] W. R. Roach and I. Balberg, Optical Induction and Detection of Fast Phase Transition in $\mathrm{VO}_{2}$, Solid State Commun. 9, 551 (1971).

[16] F. Guinneton, L. Sauques, J. C. Valmalette, F. Cros, and J. R. Gavarri, Comparative Study between Nanocrystalline Powder and Thin Film of Vanadium Dioxide $\mathrm{VO}_{2}$ : Electrical and Infrared Properties, J. Phys. Chem. Solids 62, 1229 (2001).

[17] F. Guinneton, L. Sauques, J.-C. Valmalette, F. Cros, and J.-R. Gavarri, Optimized Infrared Switching Properties in Thermochromic Vanadium Dioxide Thin Films: Role of Deposition Process and Microstructure, Thin Solid Films 446, 287 (2004).

[18] R. Li Voti, M. C. Larciprete, G. Leahu, C. Sibilia, and M. Bertolotti, Optimization of Thermochromic $\mathrm{VO}_{2}$ Based Structures with Tunable Thermal Emissivity, J. Appl. Phys. 112, 034305 (2012).

[19] A. J. Topping, U.S. Patent No. 0155154 (2004).

[20] T. Mitsuishi, On the Phase Transformation of $\mathrm{VO}_{2}$, Jpn. J. Appl. Phys. 6, 1060 (1967).

[21] K. Nagashima, T. Yanagida, H. Tanaka, and T. Kawai, Stress Relaxation Effect on Transport Properties of Strained Vanadium Dioxide Epitaxial Thin Films, Phys. Rev. B 74, 172106 (2006).

[22] C. Ko and S. Ramanathan, Stability of Electrical Switching Properties in Vanadium Dioxide Thin Films under Multiple Thermal Cycles across the Phase Transition Boundary, J. Appl. Phys. 104, 086105 (2008).

[23] N. Engheta and R.W. Ziolkowski, Electromagnetic Metamaterials: Physics and Engineering Explorations (Wiley-Interscience, Hoboken, NJ, 2006).

[24] H.S. Choi, J.S. Ahn, J.H. Jung, T.W. Noh, and D. H. Kim, Mid-infrared Properties of a $\mathrm{VO}_{2}$ Film near the Metal-Insulator Transition, Phys. Rev. B 54, 4621 (1996).

[25] M. M. Qazilbash, M. Brehm, B.-G. Chae, P.-C. Ho, G. O. Andreev, B.-J. Kim, S.J. Yun, A. V. Balatsky, M. B. 
Maple, F. Keilmann, H.-T. Kim, and D. N. Basov, Mott Transition in $\mathrm{VO}_{2}$ Revealed by Infrared Spectroscopy and Nano-imaging, Science 318, 1750 (2007).

[26] M. A. Kats, D. Sharma, J. Lin, P. Genevet, R. Blanchard, Z. Yang, M. M. Qazilbash, D. N. Basov, S. Ramanathan, and F. Capasso, Ultra-thin Perfect Absorber Employing a Tunable Phase Change Material, Appl. Phys. Lett. 101, 221101 (2012).

[27] M. A. Kats, R. Blanchard, P. Genevet, and F. Capasso, Nanometer Optical Coatings Based on Strong Interference Effects in Highly Absorbing Media, Nat. Mater. 12, 20 (2012).

[28] H. Dotan, O. Kfir, E. Sharlin, O. Blanck, M. Gross, I. Dumchin, G. Ankonina, and A. Rothschild, Resonant Light Trapping in Ultrathin Films for Water Splitting, Nat. Mater. 12, 158 (2012).

[29] M. A. Kats, S. J. Byrnes, R. Blanchard, M. Kolle, P. Genevet, J. Aizenberg, and F. Capasso, Enhancement of Absorption and Color Contrast in Ultra-thin Highly Absorbing Optical Coatings, Appl. Phys. Lett. 103, 101104 (2013).

[30] X.-L. Zhang, J.-F. Song, X.-B. Li, J. Feng, and H.-B. Sun, Anti-reflection Resonance in Distributed Bragg ReflectorsBased Ultrathin Highly Absorbing Dielectric and Its Applications in Solar Cells, Appl. Phys. Lett. 102, 103901 (2013).

[31] W. Streyer, S. Law, G. Rooney, T. Jacobs, and D. Wasserman, Strong Absorption and Selective Emission from Engineered Metals with Dielectric Coatings, Opt. Express 21, 9113 (2013).

[32] J. W. Cleary, R. Soref, and J. R. Hendrickson, Long-Wave Infrared Tunable Thin-Film Perfect Absorber Utilizing Highly Doped Silicon-on-Sapphire, Opt. Express 21, 19363 (2013).

[33] R. J. Lauf, C. Hamby, M. A. Akerman, and A. W. Trivelpiece, U.S. Patent No. 5313325 (1994).

[34] N. Gao, H. Sun, and D. Ewing, Heat Transfer to Impinging Round Jets with Triangular Tabs, Int. J. Heat Mass Transfer 46, 2557 (2003).
[35] M. H. Friedman, U.S. Patent No. 5734495 (1998).

[36] S. Shilov, "Emission Measurements" (unpublished).

[37] F. Gervais and B. Piriou, Anharmonicity in Several-PolarMode Crystals: Adjusting Phonon Self-Energy of LO and TO modes in $\mathrm{Al}_{2} \mathrm{O}_{3}$ and $\mathrm{TiO}_{2}$ to Fit Infrared Reflectivity, $\mathrm{J}$. Phys. C 7, 2374 (1974).

[38] J. Narayan and V. M. Bhosle, Phase Transition and Critical Issues in Structure-Property Correlations of Vanadium Oxide, J. Appl. Phys. 100, 103524 (2006).

[39] Y. Muraoka, Y. Ueda, and Z. Hiroi, Large Modification of the Metal-Insulator Transition Temperature in Strained $\mathrm{VO}_{2}$ Films Grown on $\mathrm{TiO}_{2}$ Substrates, J. Phys. Chem. Solids 63, 965 (2002).

[40] H. Futaki and M. Aoki, Effects of Various Doping Elements on the Transition Temperature of Vanadium Oxide Semiconductors, Jpn. J. Appl. Phys. 8, 1008 (1969).

[41] M. Nishikawa, T. Nakajima, T. Kumagai, T. Okutani, and T. Tsuchiya, Adjustment of Thermal Hysteresis in Epitaxial $\mathrm{VO}_{2}$ Films by Doping Metal Ions, J. Ceram. Soc. Jpn. 119, 577 (2011).

[42] S. Law, V. Podolskiy, and D. Wasserman, Towards Nanoscale Photonics with Micro-scale Photons: The Opportunities and Challenges of Mid-infrared Plasmonics, Nanophotonics 2, 103 (2013).

[43] T. Driscoll, H.-T. Kim, B.-G. Chae, B.-J. Kim, Y.-W. Lee, N. M. Jokerst, S. Palit, D. R. Smith, M. Di Ventra, and D. N. Basov, Memory Metamaterials, Science 325, 1518 (2009).

[44] M. A. Kats, R. Blanchard, P. Genevet, Z. Yang, M. M. Qazilbash, D. N. Basov, S. Ramanathan, and F. Capasso, Thermal Tuning of Mid-infrared Plasmonic Antenna Arrays Using a Phase Change Material, Opt. Lett. 38, 368 (2013).

[45] V. G. Golubev, V. Yu. Davydov, N. F. Kartenko, D. A. Kurdyukov, A. V. Medvedev, A. B. Pevtsov, A. V. Scherbakov, and E. B. Shadrin, Phase TransitionGoverned Opal- $\mathrm{VO}_{2}$ Photonic Crystal, Appl. Phys. Lett. 79, 2127 (2001). 